\title{
Cosmic Applications of Relative Energy between Quarks in Nucleons
}

\author{
F. C. Hoh \\ Retired, Uppsala, Sweden \\ Email: hoh@telia.com
}

How to cite this paper: Hoh, F.C. (2019) Cosmic Applications of Relative Energy between Quarks in Nucleons. Journal of Modern Physics, 10, 1645-1658. https://doi.org/10.4236/jmp.2019.1014108

Received: November 1, 2019

Accepted: November 26, 2019

Published: November 29, 2019

Copyright (C 2019 by author(s) and Scientific Research Publishing Inc. This work is licensed under the Creative Commons Attribution International License (CC BY 4.0).

http://creativecommons.org/licenses/by/4.0/

\begin{abstract}
By taking into account the relative energy between the diquark and the quark in nucleons, the gravitational singularity in a black hole created from a collapsing neutron star can be removed; compatibility with quantum mechanics is restored. This black hole becomes a "black" neutron star. The negative relative energy identified as dark matter in the previous paper can account for the galaxy rotation curve. The positive relative energy identified as dark energy in the previous paper can explain the accelerating expansion of the universe. A possible scenario for cosmic ray generation is given.
\end{abstract}

\section{Keywords}

Gravitational Singularity, Relative Energy between Quarks, Scalar Strong Interaction Hadron Theory, "Weightless" Neutron, Black Neutron Star, Galaxy Rotation Curve, Accelerating Expansion of Universe, Cosmic Ray

\section{Introduction}

A neutron star with mass greater that the Tolman-Oppenheimer-Volkoff (TOV) limit $M_{T O V} \sim 3$ solar mass $M_{S U N}$ becomes a black hole in which the star core collapses to a gravitational mass singularity in general relativity [[1] p172], [[2] black hole/\$2.3]. The quark structure of the neutrons has been neglected. But in these dense stellar objects, gravitational forces are strong and their gradients large so that such a neglect cannot be carried out without appropriate justification. Further, such a singularity contradicts quantum mechanics.

Dark matter needed to account for many observation, e. g., the galaxy rotation curve, and the dark energy required to drive the rapidly expanding universe remain hypothetical as they cannot be observed.

Recently, it was shown [3] that the "hidden", unobservable relative energy between the diquark and quark in nucleons can interact with their ambient gra- 
vitational potential and possibly play the roles of dark matter and dark energy in the universe. The underlying theory is the scalar strong interaction hadron theory SSI [4] [5].

In this paper, it is shown that, as a neutron on the surface of a neutron star with mass $\sim M_{T O V}$ falls toward the star center, not only gravitational energy is released, but a negative relative energy also emerges simultaneously. These two energies can cancel each other and prevent the collapse of the star and formation of a mass singularity. The negative relative energy generated in an expanding galaxy can play the role of dark matter and account for the galaxy rotation curve. The positive relative energy generated in outer regions of the universe can play the role of dark energy, leads to accelerating expansion of the universe and may eventually give rise to cosmic rays.

The relevant parts of SSI are outlined in Sections 2-5. Creation of negative relative energy applied to neutron star collapse is considered in Sections 6-7. In Section 8 , such negative relative energy reinforces the existing gravitational potential in an expanding galaxy to account for the galaxy rotation curve qualitatively. The accelerating expansion of the universe by means of the positive relative energy created in the outer parts of the universe is qualitatively outlined in Section 9. Some scenarios of the outer regions of the universe including cosmic ray generation are shown in Section 10. The so-obtained scenario of the universe is summarized in Section 11.

\section{Outline of Construction of Baryon Wave Equations in SSI [4] [5]}

The starting point is the Dirac equations for three quarks $A, B$ and $C$ with masses $m_{A}, m_{B}$ and $m_{C}$ located at $x_{F}, x_{I I}$ and $x_{I I I}$, respectively. The quarks interact with each other via scalar strong potentials $V_{A B}\left(x_{I}\right), V_{A C}\left(x_{I}\right), \cdots$ in [[6] (2.1-3) or [4] [5] (9.1.1-3)]. These equations are in van der Waerden's spinor form which is manifestly Lorentz covariant contrary to the conventional bispinor Dirac equation which is not. For quark $B$, for instance, the equations read

$$
\begin{gathered}
\partial_{I I}^{d \dot{e}} \chi_{B \dot{e}}\left(x_{I I}\right)-i\left(V_{B C}\left(x_{I I}\right)+V_{B A}\left(x_{I I}\right)+V_{B G}\left(x_{I I}\right)\right) \psi_{B}^{d}\left(x_{I I}\right)=i m_{B} \psi_{B}^{d}\left(x_{I I}\right) \\
\partial_{I I \dot{e} f} \psi_{B}^{f}\left(x_{I I}\right)-i\left(V_{B C}\left(x_{I I}\right)+V_{B A}\left(x_{I I}\right)+V_{B G}\left(x_{I I}\right)\right) \chi_{B \dot{e}}\left(x_{I I}\right)=i m_{B} \chi_{B \dot{e}}\left(x_{I I}\right)
\end{gathered}
$$

where $\partial_{I}=\partial / \partial x_{I}, \cdots$. The spinor indices run from 1 to 2 and the quark spinors $\chi_{B}$ and $\psi_{B}$ are linear combinations of the four components in the conventional Dirac bispinor $\psi$ according to [[4] [5] (C11)]. $m_{B}$ is quark mass, about $2 / 3$ of the proton mass [[4] [5] Table 5.2]. The scalar strong potentials $V_{B A}$ and $V_{B C}$ generated by quark $A$ and $C$, respectively, are governed by [[6] (2.4) or [4] [5] (9.1.4)]. There is no gauge fields for electromagnetic interactions between the quarks. Here, the scalar gravitational potential $V_{G B}$ from ambient matter and energy, neglected in the references, has been included.

To construct baryon wave functions, the three pairs of quark equations, one of them being (2.1), together with the cited strong potential equations, are multip- 
lied together and the products of the quark spinors and those of the strong potentials are generalized to baryon wave functions and baryon potentials nonseparable in $X_{F}, x_{I I}$ and $x_{I I I}$ according to [[4] [5] Sec. 9.2]. For ground state baryons, quark $C$ at $X_{I I I}$ has been merged into quark $A$ at $X_{I}$ to form a diquark at $X_{I}$. The nearly intractable three body problem is thus reduced to a manageable two body problem.

In [[4] [5] Sec. 9.3], each quark mass above has been generalized to a quark mass operator operatng on a flavour function in a fictitious, complex internal or flavour space $Z^{p}$, where $p$ runs from 1 to 6 representing the six quark flavours. The quark mass is recovered as eigenvalue of such an operator. The three flavour functions, one for each quark, are analogously multiplied together and generalized to a nonseparable baryon flavour function. The product of the three mass operators are similarly generalized to a nonseparable baryon mass operator in the flavour space and leads to a mass $M_{b}$ [[4] [5] (9.3.19)] representing the quark mass contribution to the baryon mass.

The baryon flavour function can now be removed leaving behind the ground state baryon wave equation [[6] (2.9)], [[4] [5] (9.3.16)]

$$
\begin{gathered}
\partial_{I}^{a \dot{b}} \partial_{I}^{g h} \partial_{I I \dot{e} f} \chi_{\{\dot{j} \dot{b}\}}^{f}\left(x_{I}, x_{I I}\right)=-i\left(M_{b}^{3}+\Phi_{b}\left(x_{I}, x_{I I}\right)\right) \psi_{\dot{e}}^{\{a g\}}\left(x_{I}, x_{I I}\right) \\
\partial_{I \dot{b} c} \partial_{I \dot{h} k} \partial_{I I}^{d \dot{d}} \psi^{\{c k\}}\left(x_{I}, x_{I I}\right)=-i\left(M_{b}^{3}+\Phi_{b}\left(x_{I}, x_{I I}\right)\right)_{\{\dot{i b j}\}}^{{ }^{d}}\left(x_{I}, x_{I I}\right) \\
M_{b}=\left(m_{A}+m_{B}+m_{C}\right) / 2
\end{gathered}
$$

The interbaryon strong potential $\Phi_{b}$ is a triple product of three strong interaction quark potentials of the type of $V_{B A}$ and $V_{B C}$ in (2.1) and is governed by [[4] [5] (9.2.13b)]. The six component $\chi$ and $\psi$ can be decomposed into a quartet or spin $3 / 2$ part, which can be separated off, leaving behind a doublet or spin $1 / 2$ part [[4] [5] (10.0.6)]:

$$
\begin{gathered}
\partial_{I}^{a \dot{b}} \partial_{I I}^{f e} \partial_{I}^{e \dot{f}} \chi_{0 \dot{b}}\left(x_{I}, x_{I I}\right)=-i 2\left(M_{b}^{3}+\Phi_{b}\left(x_{I}, x_{I I}\right)\right) \psi_{0}^{a}\left(x_{I}, x_{I I}\right) \\
\partial_{I \dot{b c}} \partial_{I I \dot{e} h} \partial_{I \dot{h} e} \psi_{0}^{c}\left(x_{I}, x_{I I}\right)=-i 2\left(M_{b}^{3}+\Phi_{b}\left(x_{I}, x_{I I}\right)\right) \chi_{0 \dot{b}}\left(x_{I}, x_{I I}\right)
\end{gathered}
$$

where $\chi_{0}$ and $\psi_{0}$ the wave functions of the doublet baryons.

\section{Laboratory and Relative Spaces}

Since quarks cannot be observed, their coordinate spaces are converted into an observable laboratory space $X^{\mu}$ for the baryon and a relative space $x$ between the diquark and the quark via the linear transformation given above [[7] (6.2)] or by [[6] (5.1)], [[4] [5] (3.1.3a)]

$$
x^{\mu}=x_{I I}^{\mu}-x_{I}^{\mu}, \quad X^{\mu}=\left(1-a_{m}\right) x_{I}^{\mu}+a_{m} x_{I I}^{\mu}
$$

For observable particles, $a_{m}$ is often determined by that $X^{\mu}$ is the center of mass of these particles. If these particles have equal mass, $a_{m}=1 / 2$. Such kind of determination cannot be done here because quarks are not observable individually. $a_{m}$ has been taken to be an arbitrary real constant and represents a new 
degree of freedom. The relative space $x=\left(x^{0}, \underline{x}\right)$ is "hidden" [[4] [5] (3.1.3)] and cannot be observed. If it were observable, then (3.1), with a given $a_{m}$, leads to that both the diquark at $X_{I}$ and quark at $X_{I I}$ become observables, contrary to experience. This can also be seen directly in the first of (3.1) in which the right side members cannot be measured; hence $x^{\mu}$ is also "hidden", independent of $a_{m}$. It turns out that the bulk of hadron physics in SSI lies in such "hidden" spaces.

The baryon wave functions in (2.1) have been factorized into the form of [[4] [5] (10.1.1)],

$$
\begin{aligned}
\chi_{0 \dot{b}}\left(x_{I}, x_{I I}\right) & =\chi_{0 \dot{b}}(\underline{x}) \exp \left(-i K_{\mu} X^{\mu}+i \omega_{K} x^{0}\right) \\
\psi_{0}^{a}\left(x_{I}, x_{I I}\right) & =\psi_{0}^{a}(\underline{x}) \exp \left(-i K_{\mu} X^{\mu}+i \omega_{K} x^{0}\right) \\
K_{\mu} & =\left(E_{K},-\underline{K}\right) \text { [[4] [5] (3.1.6)] }=
\end{aligned}
$$

where $E_{K}$ is the energy of the baryon and $\underline{K}$ its momentum. $x^{0}$ is the relative time and $-\omega_{K}$ the associated relative energy in the "hidden" relative space and can also not be observed within SSI.

In spherical coordinates, $\underline{x}=(r, \theta, \phi)$ [[4] [5] (3.1.7b)], the doublet wave functions in (3.2) with total angular momentum $j=1 / 2$ and orbital angular momentum $I=0$ read [[4] [5] (10.2.3)]

$$
\psi_{0}^{1}(\underline{x})=g_{0}(r) Y_{00}(\theta, \phi)+i f_{0}(r) \sqrt{\frac{1}{3}} Y_{10}(\theta, \phi), \quad \psi_{0}^{2}(\underline{x})=i f_{0}(r) \sqrt{\frac{2}{3}} Y_{11}(\theta, \phi)
$$

where the $Y$ s are the usual spherical harmonics. $\chi_{0 a}$ is found by changing the signs of $f_{0}(r)$ in (3.4).

\section{Radial Wave Equations in Relative Space, Solutions and Results}

Consider baryons at rest, $\underline{K}=0 . a_{m}=1 / 2$ is set as in the meson case [[4] (3.5.7)], [[5] (5.7.2)]. Similarly, the "hidden" relative energy $-\omega_{0}=0$ is also set following the meson case [[4] (3.5.6)], [[5] (5.7.1)]. Insertion of (3.1-4) into (2.3) using [[4] [5] (3.1.4)] leads to the radial wave equations [[6] (6.9)], [[4] [5] (10.2.12)]

$$
\begin{gathered}
{\left[\frac{E_{0}^{3}}{8}+M_{b}^{3}+\Phi_{b d}(r)+\frac{E_{0}}{2} \Delta_{0}\right] g_{0}(r)+\left(\frac{E_{0}^{2}}{4}+\Delta_{0}\right)\left(\frac{\partial}{\partial r}+\frac{2}{r}\right) f_{0}(r)=0} \\
{\left[\frac{E_{0}^{3}}{8}-M_{b}^{3}-\Phi_{b d}(r)+\frac{E_{0}}{2} \Delta_{1}\right] f_{0}(r)-\left(\frac{E_{0}^{2}}{4}+\Delta_{1}\right) \frac{\partial}{\partial r} g_{0}(r)=0}
\end{gathered}
$$

where the subscript $d$ denotes doublet and [[4] [5] (10.2.2a)] gives the diquark-quark strong interaction potential

$$
\Phi_{b d}(r)=\frac{d_{b}}{r}+d_{b 0}+d_{b 1} r+d_{b 2} r^{2}+d_{b 4} r^{4}
$$

here, the nonlinear potential $\Phi_{c d}(\underline{x})$ [[4] [5] (10.2.2)] vanishes for large normalization volume $\Omega_{c b} \rightarrow \infty$ in [[4] [5] (10.3.14)]. The $d_{b}$ 's are unknown integration constants.

The two coupled third order Equations (4.1) have been converted into six first 
order equations [[4] (10.7.5)], [[5] (10.4.5)] which have six linearly independent solutions each associated with its own $\lambda_{+}$values in [[4] [5] (10.2 8a)] found from the sixth order indicial equation [[5] (10.4.4)]. These eventually led to two free parameters $w_{(1)}$ and $w_{(2)}$ in [[4] [5] (11.1.1)]. It turned out that only $d_{b 4}=0$ led to results in near agreement with data [[5] \$11.1.3]. Thus, there are four $d_{b}$ and two $w$ totalling six constants that can be varied to satisfy the six boundary conditions posed by the six first order system.

Due to the large number of unknown constants, (4.1-2) could not be solved as a conventional eigenvalue problem. A less ambitious approach has been adopted. The known mass of the neutron is used as input for the eigenvalue $E_{0}$ and the quark masses obtained from meson spectra given in [[4] [5] Table 5.2] are used as input for $M_{b}$ according to (2.2c) where $m_{A}=m_{C}$ is the $d$ quarks mass and $m_{B}$ the $u$ quark mass. The six first order equations [[4] (10.7.5)], [[5] (10.4.5)] have been solved on a computer. It turned out that given a $d_{b 2}$ value, the remaining five constants can be freely chosen such that $g_{0}(r)$ and $f_{0}(r)$ converge at large $r$. This is allowed since this takes place in the "hidden" relative space.

Here, the word "finite" on the first line of the second paragraph on [[5] p239] turned out to be incorrect in subsequent numerical calculations and should be changed to "infinite"; $d_{b 2}$ can be varied continuously to yield converging $g_{0}(r)$ and $f_{0}(r)$. One so-obtained neutron wave functions are plotted in [[4] Figure 12.1], [[5] Figure 11.1b] reproduced in Figure 1.

These wave functions have led to the nearly correct predictions of the neutron life and the electron asymmetry parameter $A$ or the neutrino asymmetry parameter $B[[5]$ Table 12.1] in its beta decay.

\section{Relative Energy and Gravitation}

If $a_{m}=1 / 2$ and $-\omega_{0}=0$ used in (4.1) were not assumed, a derivative in (2.3) operated on (3.2) will, in terms of (3.1) [see [4] [5] (3.1.4)], be changed as follows

$$
\begin{aligned}
\partial_{I}^{a \dot{b}} & =\left(1-a_{m}\right) \partial_{X}^{a \dot{b}}-\partial^{a \dot{b}}=\left(1-a_{m}\right)\left(-\delta^{a \dot{b}} \partial_{X 0}-\underline{\sigma}^{a \dot{b}} \partial_{\underline{X}}\right)+\delta^{a \dot{b}} \partial_{0}+\underline{\sigma}^{a \dot{b}} \underline{\partial} \\
& =\delta^{a \dot{b}} i \frac{1}{2} E_{0}+\underline{\sigma}^{a \dot{b}} \underline{\partial} \rightarrow \delta^{a \dot{b}} i\left(\left(1-a_{m}\right) E_{0}+\omega_{0}\right)+\underline{\sigma}^{a \dot{b}} \underline{\partial}
\end{aligned}
$$

The expressions on both sides of the arrow will be equal if [[7] (6.6) or [4] [5] (3.1.10a)]

$$
a_{m}=\frac{1}{2}+\frac{\omega_{0}}{E_{0}}
$$

which keeps (4.1) invariant. The relative energy $-\omega_{0}$ is "hidden" with respect to strong and electromagnetic but not gravitational interactions [3]. This is indicated by $V_{B G}$ in (2.1), which has been neglected in SSI because it is generally small next to the strong $V_{B A}, V_{B C}$ there. In neutron stars and black holes, however, $V_{B G}$ can be large and may not be neglected. Since $a_{m}$, hence also $\omega_{0}$, can take on any real value, from $-\infty$ to $+\infty$, the relative energy can be important is astrophysics. 


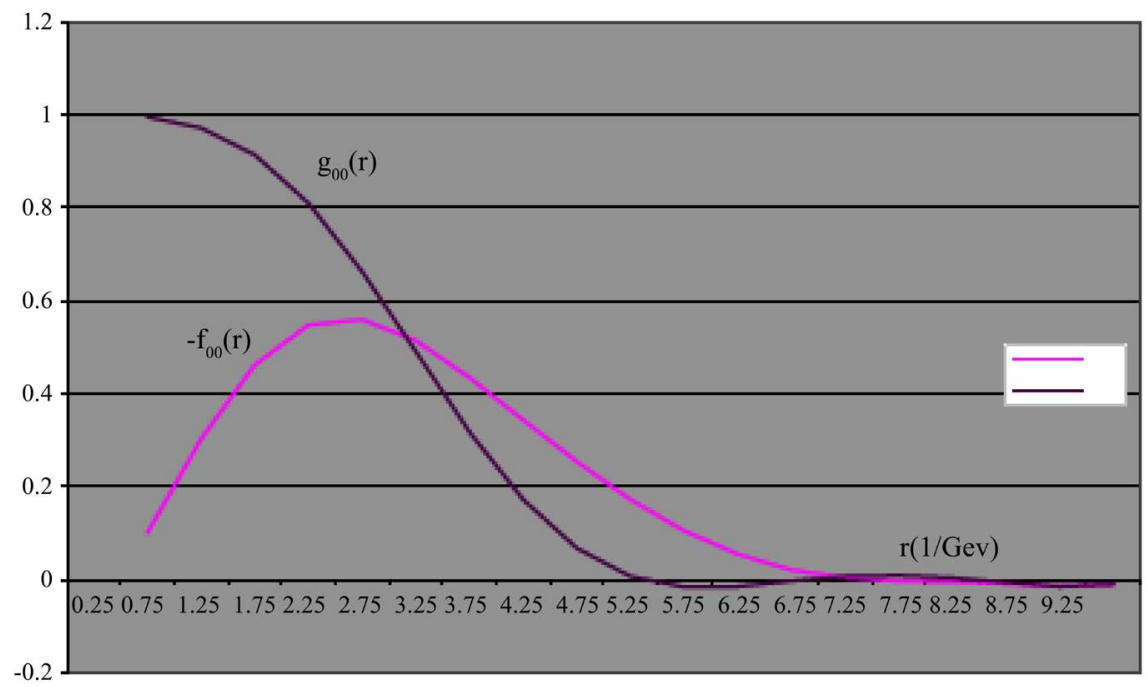

Figure 1. $g_{00}(r)$ and $f_{00}(r)$ are $g_{0}(r)$ and $f_{0}(r)$ in (4.1) normalized according to [[4] [5] (10.3.14)] for the $d_{b 2}=-0.3202$ case in [[4] [5] Table 11.1] where the four $d_{b}$ and two $w$ constants are given. $r$ is the diquark-quark distance. This wave function has been used to evaluate neutron decay parameters in [[5] \$12.6.3].

In [3], $-\omega_{0}>0$ is identified as dark energy and $-\omega_{0}<0$ plays the role of dark matter. Both $E_{0}$ and $\omega_{0}$ are observables with respect to gravitational forces. Lorentz invariance leads to that the associated baryon momentum $\underline{K}$ in (3.3) and the relative momentum represented by $\underline{\partial}$ in (5.1) are also observables. Thus, the conjugate variables $\underline{X}$, the coordinate of the baryon, and $\underline{X}$, the relative coordinate in (3.1), are also observables, remembering that $\underline{x}$ is still a "hidden" variable with respect to strong forces. By (3.1), both the diquark coordinate $\underline{x}_{I}$ and quark coordinate $\underline{x}_{I I}$ are now observables with respect to gravitation indicated by $V_{B G}$ in (2.1).

\section{Neutron Star Collapse Scenario}

Consider the following idealized scenario. A neutron star with a mass $M_{N S}=$ $M_{N S_{a}}$ a critical mass, such that the quantum degeneracy pressure of the neutrons and the strong neutron-neutron repulsion precisely balance off the gravitational pressure. Let an external neutron arrive at the surface of this star. The star now becomes too heavy and a gravitational collapse is anticipated to start. Conventionally, this collapse gives rise to a black hole and continues until the star ends up in a mass singularity at its center; the quark structure of the neutron is ignored. Actually, in neutron stars and black holes, the gravitational forces are strong and their gradients large that the diquark-quark structure of the neutron may need be taken into account. This scenario is illustrated in Figure 2.

In Figure 2, an external neutron arrives on the surface of the neutron star at position $a$ on the right part of the figure. The interquark distance is $x_{I I, a}-x_{I, a}=r_{a} \sim 4 \mathrm{fm}$ according to Figure 1. $X_{a}$ lies in the middle corresponding to $a_{m}=1 / 2$ in (3.1) and (4.1) and hence $\omega_{0}=0$ by (5.2). This neutron falls from $X_{a}$ to $X_{b}$. To begin with, the three positions $X_{I, a}, X_{a}$ and $X_{I I, a}$ are simply 


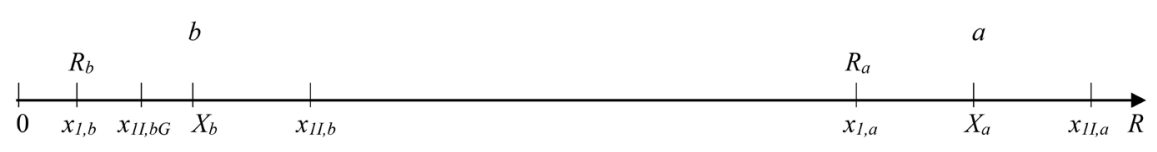

Figure 2. Illustration of the above idealized scenario. The horizontal line represents the radius $R$ inside the neutron star. The center of the star is at $R=0$. An external neutron arrives at $X_{a}$ on the surface of this star at $R=R_{a}=X_{I, a}$ in position $a$ and falls to $X_{b}$ closer to the star center in position $b . X_{I, a}$ and $x_{I I, a}$ are the positions of the diquark and quark of the arriving neutron, respectively. When this neutron has moved to $X_{b}$, the diquark and quark positions become at first $x_{I, b}$ and $X_{I I, b}$, respectively; the diquark-quark distance is the same in both positions. Due to gravitational collapse, however, the neutron star becomes smaller at position $X_{b}$ so that the diquark-quark distance in the arriving neutron is also reduced via the replacement of $x_{I I, b}$ by $x_{I, b G} R_{b}=x_{I, b}$ is radius of the collapsed star when the neutron has moved to $X_{b}$.

shifted to the left to become $x_{I, b}, X_{b}$ and $x_{I, b}$, respectively. The diquark-quark distance $r_{b}=x_{I I, b}-x_{I, b}=r_{a}$ remains unchanged. In the same fall, however, the neutron star radius at position $b$ is reduced to $R_{b}<R_{q}$ due to gravitational collapse. The incoming neutron is thus similarly compressed to become smaller by a factor of $R_{b} / R_{a}$ so that $r_{b}$ is also reduced to a smaller value $r_{b G}$ by the same factor;

$$
r_{b}=x_{I I, b}-x_{I, b} \rightarrow r_{b G}=x_{I I, b G}-x_{I, b}=r_{a} \alpha, \quad \alpha=R_{b} / R_{a}<1
$$

As was mentioned at the end of Section 5, gravity interacts directly with the quark at $x_{I I}$ in (2.1), hence also with that in Figure 2. Phenomenologically, it also interacts with the diquark at $x_{I}$ in Figure 2. An equation of motion for the diquark on par with (2.1) for the quark has not been constructed. In an eventual such equation, the diquark mass is expected to be about twice the mass of the quark in (2.1) or at least greater than it. Assuming this, gravity will pull the diquark closer to the star center relative to the quark, as is indicated in Figure 2. Note that the quark and the diquark are point particles in quantum theory and hence cannot be "spaghettified" here. The radius $R_{b}$ of the star at position $b$ is taken to be $X_{I, b}$

If $X_{b}$ were taken to be the star radius $R_{b}$ at position $b$, then the diquark coordinate $X_{p b}$ in Figure 2 would be negative at the star center $X_{b}=0$, contrary to the requirement that a radius $\geq 0$. Practically, $X_{b}$ and $R_{b}$ are of the magnitude km while their difference is of magnitude fm; both are nearly the same.

The radial wave Equations (4.1-2) hold for the arriving neutron in position $a$. At position $b$, (6.1) shows that the diquark-quark distance or radius $r$ in (4.1) is reduced by a factor of $\alpha$. Equations (4.1-2) remain invariant under the transformations $r \rightarrow r \alpha, E_{0} \rightarrow E_{0} / \alpha, M_{b} \rightarrow M_{b} / \alpha, d_{b 2} \rightarrow d_{b 2} / \alpha^{5}, \cdots$. The neutron mass $E_{0}$ and the quark masses in $M_{b}$ at position $b$ are unchanged. So does also the confining constant $d_{b 2}=-0.3202$ in Figure 1 ; this value is tied up by neutron decay [[5] \$12.6.3]. The remaining three $d_{b}$ and two $w$ constants mentioned above Figure 1 can be varied to produce converging $g_{0}(\alpha r)$ and $f_{0}(\alpha r)$ analogous to the computations that led to Figure 1. These wave functions will obviously be much narrower than those in Figure 1 having an average width of 
$\alpha r_{a} \sim 4 \alpha \mathrm{fm}$.

\section{Removal of Mass Singularity and "Black" Neutron Star}

Application of (3.1), (5.2) and (6.1) to position $b$ in Figure 1 yields

$$
a_{m}=\frac{1}{2}+\frac{\omega_{0}}{E_{0}}=1+\frac{X_{b}-x_{I I, b G}}{x_{I I, b G}-x_{I, b}}=\frac{1}{2} \frac{r_{a}}{r_{b G}}=\frac{R_{a}}{2 R_{b}}, \quad-\frac{\omega_{0}}{E_{0}}=-\frac{R_{a}}{2}\left(\frac{1}{R_{b}}-\frac{1}{R_{a}}\right)
$$

where $-\omega_{0}$ is the relative energy gained when the external neutron arriving at position $a$ falls to position $b$. In this fall, the gravitational energy $E_{G}$ released is given by

$$
\frac{E_{G}}{E_{0}}=\frac{R_{S a}}{2}\left(\frac{1}{R_{b}}-\frac{1}{R_{a}}\right), \quad R_{S a}=2 G M_{N S a}
$$

where $G$ is the gravitational constant and $R_{S a}$ is the Schwarzschild radius of this collapsing star with radius $R_{a}$ in Figure 2 . These two energies cancel each other when the star's radius

$$
R_{a}=R_{S a}
$$

i.e., when the neutron star in Figure 2 with the critical mass $M_{N S a}$ has a radius $R_{a}$ equal to its Schwarzschild radius $R_{S a}$. At the star center $R_{b}=0$, both energies become infinite but these both singularities cancel; no net mass singularity is created when (7.3) holds. This is consistent with that SSI is a quantum theory not allowing such a singularity. Note that when approaching the star center and $R_{b}$ becomes of the size of the neutron of a few fm. For $R_{a} \sim 10 \mathrm{~km} ; a_{m}=R_{a} / 2 R_{b}$ is a huge number $\sim 10^{18}$.

In this case, the fall of this external neutron actually gains no energy because $E_{G}-\omega_{0}=0$ for all $R_{b}$. This neutron therefore remains at position $a$ and becomes "weightless". The anticipated collapse of the neutron star does not start and no mass singularity is created.

The radius $R_{a}$ of the initial neutron star has been estimated by equating gravitational pressure to the pressure of the degenerate neutrons [[1] p161]

$$
R_{a}=0.0026 R_{\text {Earth }}\left(\frac{M_{S U N}}{M_{N S a}}\right)^{1 / 3}
$$

which together with (7.2-3) yields $R_{a}=R_{S a}=10.8 \mathrm{~km}$ and $M_{N S a}=3.6 M_{S U N}$. This mass is consistent with the similarly estimated maximum mass $4.3 M_{S U N}$ [[1] p162] of a neutron star. It is of somewhat higher than $M_{T O V} \sim 3 M_{S U N}$. cited in Section 1 . However, estimates of $M_{T O V}$ are uncertain and vary widely due to that the equations of state for extremely dense matter are not well known and to uncertain effects of neutron-neutron repulsion and of pressure in general relativity [[1] p162]. Thus, the so-estimated star mass may be used on equal footing with those in the literature.

A possible scenario is as follows. As additional neutrons arrive at the star surface, a thin shell of weightless neutrons is added to the star at first. Let the mass 
of this shell be $\Delta M_{N S a}$, the new Schwarzschild radius will be $R_{S a \Delta}=(1+\Delta) R_{S a}$ and the new star radius will be $R_{a \Delta}=(1+\Delta)^{1 / 3} R_{a}$, assuming that the neutron density in the shell is the same as that in the star.

Since $R_{S a \Delta}>R_{a \Delta}$, a black hole is created. As a new neutron arrive at the new star surface with radius $R_{a}$, it will tend to fall inwards. This time, the gravitational energy (7.2) will slightly exceed the relative energy loss (7.1) and this new neutron tends to fall slowly. However, the pressure of the degenerate neutron gas in the shell is now unopposed by the weightless neutrons and this gas will therefore expand accompanied by a reduced density. If the expansion reaches a radius $>R_{S a \Delta}$, the back hole is lost and this enlarged star will be a visible neutron star similar to the initial star in Figure 2. If the expansion stops just inside $R_{S a \Delta}$, the created black hole persists and one arrives at a situation nearly the same as that on the right part of Figure 2 with $R_{a}$ replaced by $R_{a \Delta}$ and (7.3) holds. This process may be repeated and more shells are added. It can in principle lead to a super-massive black hole. How the neutrons, part of them "weightless", are distributed inside such a black hole is unknown and needs not be known, inasmuch as they are inside its Schwarzschild radius and cannot be observed any way; there is no conflict with observation.

In this scenario, the neutrons in such a black hole fill it up to its Schwarzschild radius. Such a black hole may more suitably be called a "black" neutron star. The absence of mass singularity here is consistent with the conjecture that an eventual future quantum gravity theory will not contain any singularity.

\section{Galaxy Rotation Curve without Dark Matter}

There is a large body of data that require the presence of dark matter. The first one is the galaxy rotation curve [[2] Dark Matter \$3.1]. However, nothing is known about this hypothetical dark matter. In [3], this matter has been identified as the negative relative energy $-\omega_{0}<0$ between the diquark and quark in nucleon. Here, the role of the dark matter in accounting for this rotation curve will be played by this $-\omega_{0}$.

Consider again an idealized scenario as follows. A spiral galaxy with an appreciable part of its mass consisting of hydrogen gas was in its earlier stage of development. In that stage, this galaxy was smaller, denser and hotter according to the big bang model and the gravitational potential resisting such an expansion was insufficient. It therefore expanded. The situation is analogous to a violation of Jeans criterion for a gas cloud.

To illustrate the mechanism, let us turn off the gravity for a moment. Follow now the movement of a proton, denoted by $c$, in a hydrogen molecule. In this thermal expansion, this molecule will collide with other molecules, exchange energy and momentum with them and end up in a new position, labeled $d$, farther out from the galaxy center. In this journey, the forces involved are all Coulomb forces, between the proton and the orbiting electron and between the orbiting electrons in other molecules. The protons get dragged along; their quark 
structure is not involved in the expansion.

The situation is illustrated in Figure 3.

In Figure 3, the coordinate of proton $c$ is $X_{c}$ which lies in the middle between the diquark at $x_{1, c}$ and quark at $x_{I 1, c}$ This corresponds to $a_{m}=1 / 2$ and $\omega_{0}=0$ in (7.1) for a free baryon at rest, similar to those for the neutron in position $a$ in Figure 2. In this thermal expansion, these three coordinates are simply shifted to the right with $X_{c} \rightarrow X_{d}$. The new $x_{I, c}$ and $x_{I I, c}$ are on both sides of $X_{d}$ with the same separation distance.

Turn now on gravity, the situation is reversed. Gravitational effects on electrons are negligible due to their small mass. Gravitational forces now act on this proton at $X_{d}$ and try to pull it back together with other protons acted upon. However, they turn out to be too small to account for the galaxy rotation curve and prevent the escape of the outer stars. Here, such escapes are prevented by including the negative energies generated by differentiated gravitational pull on quarks and proton.

Just like the neutron star case mentioned below (6.1), gravity also acts directly on the quarks and tends to pull them towards the galaxy center, with the heavier diquark at $x_{I, d}$ closer to this center than does the lighter quark at $x_{I I, d}$ as is shown in Figure 3. Here, the diquark-quark distance $x_{I I, c}-x_{I, c}=x_{I I, d}-x_{I, d}=r_{a} \approx 4$ $\mathrm{fm}$ as was mentioned below Figure 2. This distance is fixed by the strong interquark potential (4.2) and is unaffected by gravity here; the core collapse situation in position $b$ of Figure 2 is absent here.

Applying (3.1) the gravity pulled position $d$ in Figure 3 yields the relative energy $-\omega_{0}$ given by

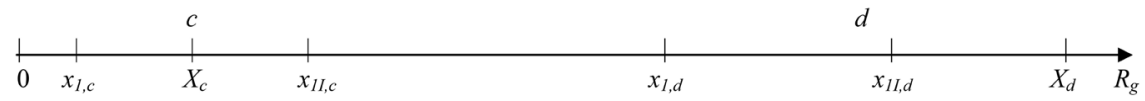

Figure 3. Illustration of the expanding galaxy scenario. The horizontal line represents the radius $R_{g}$ inside the galaxy. The center of the galaxy is at $R_{g}=0$. A proton in position $c$ is moved to position $d$ in the expansion. $X_{c}$ denotes the position of the proton at position $c$, $X_{I, c}$ that of the diquark in this proton, and $x_{I I, C}$ that of the quark. In the expansion, this proton $c$ moves from $X_{c}$ to $X_{d}$ in position $d$. When gravity is turned on, the quark coordinates become $X_{I, d}$ and $X_{I I, d}$ respectively.

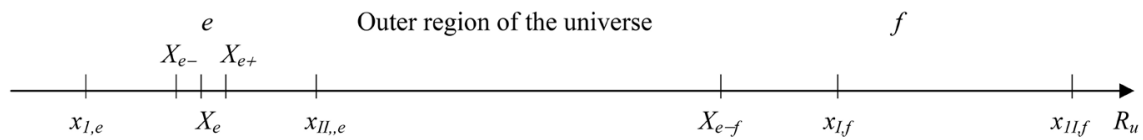

Figure 4. Illustration of the expanding universe scenario. The horizontal line represents the distance $R_{u}$ from some unspecified inner region of the universe. $X_{e}$ denotes the coordinate of a proton in a hydrogen molecule at position $e, x_{I, e}$ that of the diquark in this proton and $x_{1, e}$ that of the quark. $X_{e}$ lies in the middle between them. A second hydrogen molecule containing a second proton with its diquark and quark at the same $x_{I, e}$ and $x_{I I, e}$ is also present. The coordinate of this second proton may be $X_{e^{-}}$or $X_{e+}$ close to $X_{e^{\text {. }}}$ The second proton at $X_{e-}$ will generate a positive relative energy that pushes its both quarks outwards. The coordinate of this proton lags behind. This process continues and this second proton arrives at position $f$ with the coordinates $X_{I, f}$ and $x_{I I, f}$ for the quarks and $X_{e-f}$ for this second proton. 


$$
a_{m}=\frac{1}{2}+\frac{\omega_{0}}{E_{0}}=\frac{X_{d}-x_{I, d}}{x_{I I, d}-x_{I, d}}, \quad-\frac{\omega_{0}}{E_{0}}=\frac{1}{2}-\frac{X_{d}-x_{I, d}}{r_{a}}, \quad r_{a}=x_{I I, d}-x_{I, d} \approx 4 \mathrm{fm}(8.1)
$$

This energy has the same negative sign as the gravitational potential energy produced by matter inside $X_{d}$ and hence reinforces it to become large enough to keep the outer stars of the galaxy from escaping. It is due to the lag of the "hidden" quark coordinates $x_{I, d}, x_{I I, d}$ behind the observable proton coordinate $X_{d}$ in Figure 3, caused by gravitational pull, in the expansion. This lag is caused by that the mass of the diquark and the quark is greater than the proton mass $E_{0}$ itself, as can be seen below (2.1) and (6.1); the unobservable quarks experience greater gravitational pull than does the proton containing them. The difference between these two masses is taken up by the strong, confining potential $\Phi_{b d}(r)$ of (4.2) in the "hidden", relative space.

The required energy is $-\omega_{0} \approx-5.5 E_{0}$ per proton, when averaged over the whole universe, and is generated for "free" at "no cost". This $-\omega_{0}$ value leads to $a_{m} \approx 6$ ([3] \$6.1) so that the "whole" size of this gravitationally polarized proton in Figure 3 becomes $X_{d}-X_{I, d} \approx 24 \mathrm{fm}$ by (8.1). Further, the proton in a hydrogen atom moves in an orbit around the center of gravity of the electron-proton system. This orbit has a diameter $\approx 60 \mathrm{fm}$ so that $X_{d}$ is uncertain by this amount which is greater than the above $24 \mathrm{fm}$ in Figure 3; there will be no observational difference.

This scenario, characterized by Figure 3, is also expected to have dominated in the earlier stages of the universe when it was smaller, hotter, denser, and fast expanding.

\section{Accelerating Expansion of the Universe without Dark Energy}

The observed accelerating expansion of the universe is currently considered to be due to assumed dark energy in the outer regions of the universe. This hypothetical energy may here be replaced by the positive relative energy $-\omega_{0}>0$ corresponding to $a_{m}<1 / 2$ in (8.1).

As in Sections 6 and 8, consider the following idealized scenario. The expansion mechanism in Section 8 applied to a galaxy can analogously be used in some later stage of the development of the universe. In the outer part of the universe, its expansion leads to that the hydrogen gas density decreases and the gas temperature drops there. The expansion nearly comes to a halt. In this region, the gas is tenuous, cold and experiences very weak gravitational force.

Consider a proton in a hydrogen molecule of this gas. The configuration of this proton in position $d$ of Figure 3 , with the galaxy replaced by the universe, has now largely returned to its original form in position $c$ of Figure 3 . This proton is also similar to the neutron in position $a$ of Figure 2 with $a_{m} \approx 1 / 2$ and the pulling energy $-\omega_{0}<0$ approaching $-\omega_{0} \approx 0$. The situation is illustrated in position $e$ of Figure 4.

Let a second hydrogen molecule arrive at position $e$ simultaneously. It con- 
tains a second proton with its diquark and quark at the same $x_{I, e}$ and $x_{I I, e}$. Due to differences in the paths of these both molecules before they reach position $e$, this second proton may end up at nearby $X_{e^{-}}=X_{e}-\Delta\left(x_{I I, e}-x_{I, e}\right)$ or $X_{e+}=X_{e}+\Delta\left(x_{I I, e}-x_{1, e}\right)$ where $\Delta \ll 1$.

If its position is $X_{e+}$, then (8.1) yields a negative relative energy $-\omega_{0}=-\Delta E_{0}<0$. Analogous to the situation below (8.1), this small energy tends to pull the quarks toward the left in Figure 4 into the inner region of the universe. This tendency is countered by the slightly higher gas pressure inside $X_{e}$. No appreciable net movement of the quarks is expected; the situation is stable.

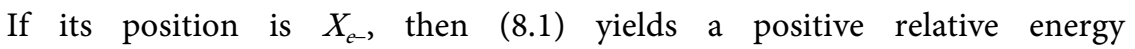
$-\omega_{0}=-\Delta E_{0}>0$. Contrary to the above $X_{e+}$ case, this small energy tends to push the quarks outward, towards the right part of Figure 4. This tendency is reinforced by the slightly higher gas pressure inside $X_{e}$; $\Delta$ increases and $a_{m}$ decreases. The quarks move toward the outer region of the universe but the observable proton coordinate $X_{e^{-}}$lags behind; this situation is opposite to that for $X_{e^{+}}$above or to that in position $d$ in Figure 3 mentioned below (8.1). After some time, the unobservable quarks arrive at $x_{I, f}$ and $x_{I I, f}$ in Figure 4. Application of (8.1) to the coordinates in position $f$ indicates that $a_{m}$ has decreased from $1 / 2-\Delta$ at position $e$ to $<0$ at position $f$ so that the positive relative energy $-\omega_{0}$ by $(8.1)$ has grown appreciably to $>E_{0} / 2$. This new, larger positive relative energy further pushes the quarks outwards at a greater pace and tends to increase the lag of the proton coordinate $X_{e-f}$ behind the quark coordinates $x_{I, f} x_{I I, f}$ in Figure 4 . This by (8.1) generates still greater $-\omega_{0}>0$ which gives still greater pushing force on the quarks. This is an unstable, "run away" situation which can in principle continue forever. The driving relative energy is "free", "costs nothing" and is not conserved. If this mechanism is applied to all protons in the outer regions of the universe, an acceleratingly expanding universe emerges.

Current data show that dark energy exceeds the energy of ordinary matter by a factor of $\approx 14$ when averaged over the universe. Identifying this energy with $-\omega_{0}$, (8.1) leads to $a_{m}=-13.5$ ([3] $\left.\$ 6.1\right)$. This value and (8.1) give the "whole" size of this second proton at position $f$ in Figure $4 x_{I I, f}-X_{e-f}=58 \mathrm{fm}$ which is close to but still within the error margin $\approx 60 \mathrm{fm}$ for $X_{e-f}$ mentioned at the end of Section 8. This margin is small relative to the size of hydrogen atom and does not alter its behavior as a hydrogen atom.

This scenario, characterized by Figure 4, also dominates in the later stages of the universe when it has become large.

\section{Scenarios in the Outer Regions of the Uiniverse}

\subsection{Possible Plasma Creation}

As the above "run way" situation continues, the "whole" size of this proton, $x_{I I, f}$ - $X_{e-f}$ in Figure 4 or the proton-quark separation gets larger and eventually reaches $0.55 \AA$, the radius of hydrogen atom. Since the observable proton coordinate $X_{e-f}$ is located at the center of the atom, the quarks will in the same orbit 
as the electron. These two objects may come to be close to each other and interact via some so far unspecified mechanism. Since the electron is much lighter than a quark, which has a mass of about $2 / 3$ of the proton mass mentioned below (2.1), it will be assumed that the electron gets ejected, noting that the ionization energy of hydrogen atom is negligible relative to the energy scale involved here. This hydrogen atom becomes ionized.

The relative energy corresponding to this case is by $(8.1)-\omega_{0} \approx 1.29 \times 10^{13} \mathrm{eV}$. In this scenario, hydrogen atoms in the expanding outer regions of the universe having this energy or greater turn into plasma.

\subsection{Generation of Cosmic Rays}

According to 10.1., the hydrogen gas in outer regions of the universe can expand until an average atom acquires an energy $\sim 1.29 \times 10^{13} \mathrm{eV}$, beyond which the atom becomes ionized. For atoms carrying higher energies, the gas becomes a tenuous plasma in intergalactic space. In this plasma, the protons continue to gain energy by the increasing $-\omega_{0}$ but the ejected electrons lag behind, inasmuch as gravity is absent in Dirac's equation for an electron in a Coulomb field. This leads to a proton current flowing radially outwards which in its turn generates transverse magnetic field. As there are inhomogeneities in gas distribution in this region, the magnetic field will also vary in space.

As the "run away" expansion in Section 9 continues, some protons become very energetic. The magnetic field will cause them to move perpendicular to their path and to the direction of the magnetic field. Some of these high energy protons may be moved by magnetic fields such that they return to the inner part of the universe. Such protons can be a source of cosmic rays with energy $>10^{13}$ $\mathrm{eV}$,

For a cosmic ray proton having a high energy of, say, $10^{20} \mathrm{eV}$, The "whole" size of this proton $x_{I J, f}-X_{e-f}$ in Figure 4 given by (8.1) is still rather small, $\sim 0.4$ $\mathrm{mm}$ (unobservable), which is hardly detectable even if it were observable.

\subsection{Diquark-Quark "Flip" and Magnetic Curtailment}

In Figures 2-4, the diquark is closer to the center of the star, galaxy or universe; $x_{I}<x_{I r}$. This is due to that the heavier diquark experiences a stronger pull towards the centers than does the lighter quark. This pulling force becomes very weak far out in the universe where the positive relative energy increases by the above run away instability pushing the quarks outwards with ever increasing proton energy and greater proton-quark separation or the "whole" size of the proton, $X_{1 I, f}-X_{e-f}$ in Figure 4.

This may in principle go on forever. This pushing force is stronger on the heavier diquark than that on the quark and may eventually push the diquark past the quark farther out and "flip" their positions from $x_{I, f}<x_{I I, f}$ in Figure 4 to $x_{I}>x_{I I}$ farther away. In this scenario, (8.1) shows that $a_{m}$, hence also $-\omega_{0}$, changes sign; the expansion force switches to contracting force. The expansion 
will slow down and change to contraction. If such processes continue, an "oscillating" scenario of the outer regions of the universe may emerge.

But even before this scenario, another one may take place. The protons with energy $>10^{13} \mathrm{eV}$ are part of a plasma with transverse magnetic field in $\$ 10.2$. Some of them will eventually move in a direction perpendicular to the direction of $R_{u}$ in Figure 4. In that motion, the acceleration mechanism in Section 9 is no longer active. In this scenario, infinite proton energy and infinite expansion of the universe's outer regions may be curtailed by the magnetic fields.

\section{Summary-Scenario of the Universe}

The present results lead to the following scenario for the universe.

1) The universe contains neither dark matter nor dark energy.

2) A heavy neutron star with mass $=M_{T O V}$ and radius $=$ its Schwarzschild radius does not collapse into a mass singularity and may be called a "black" neutron star. If every neutron star gets heavier, becomes a black hole and passes through this stage in its development, there will be no mass singularity in the universe, in agreement with that quantum mechanics does not allow such a singularity.

3) The run away instability in Section 9 provides a mechanism for an accelerating expansion of the universe. The driving positive relative energy is "free" and "costs nothing". The density and temperature of the universe will decrease with time. This scenario may in principle go on forever.

4) In outer parts of the universe, fast expanding hydrogen gas may turn into plasma and part of it may become cosmic rays. There may also exist a scenario in which the above free expansion can halt and eventually reverts to contraction.

The above results are derived phenomenologically by joining SSI to aspects of general relativity. A formal integration of these both theories is beyond reach; no quantum gravity theory exists presently.

\section{Conflicts of Interest}

The author declares no conflicts of interest regarding the publication of this paper.

\section{References}

[1] Ryan, S.G. and Norton, A.J. (2010) Stellar Evolution and Nucleosynthesis. Cambridge University Press, Cambridge.

[2] Wikipedia (2019). https://en.wikipedia.org/wiki/Main_Page

[3] Hoh, F.C. (2019) Journal of Modern Physics, 10, 635.

[4] Hoh, F.C. (2011) Scalar Strong Interaction Hadron Theory. Nova Science Publishers, New York.

[5] Hoh, F.C. (2019) Scalar Strong Interaction Hadron Theory. II Nova Science Publishers, New York.

[6] Hoh, F.C. (1994) International Journal of Theoretical Physics, 33, 2125.

[7] Hoh, F.C. (1993) International Journal of Theoretical Physics, 32, 111. 